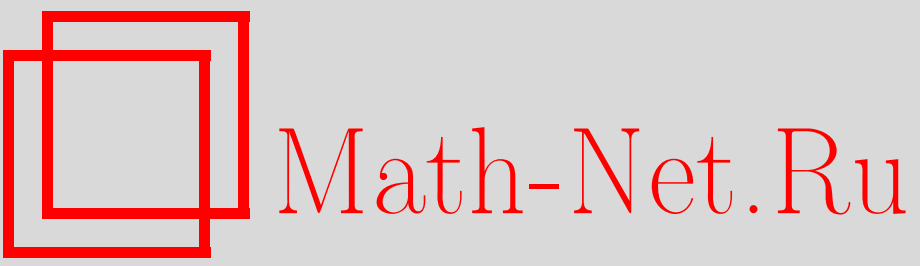

С. С. Рышков, О структуре примитивного параллелоэдра и о последней проблеме Вороного, УМН, 1998, том 53, выпуск 2, 161-162

DOI: https://doi.org/10.4213/rm44

Использование Общероссийского математического портала Math-Net.Ru подразумевает, что вы прочитали и согласны с пользовательским соглашением

http://www.mathnet.ru/rus/agreement

Параметры загрузки:

IP: 18.208 .226 .222

26 апреля 2023 г., 18:23:33 


\title{
О СТРУКТУРЕ ПРИМИТИВНОГО ПАРАЛЛЕЛОЭДРА И О ПОСЛЕДНЕЙ ПРОБЛЕМЕ ВОРОНОГО
}

\author{
C. С. Рышков
}

В заметке дается решение двух проблем теории $n$-мерных параллелоэдров (далее "ПалЭ"), во-первых, (в п. $\left.1^{0}\right)$ изучение примитивных ПалЭ сводится к исследованию ПалЭ нового класса “коренных ПалЭ”, примеры таких ПалЭ приводятся $\left(2^{0}-4^{0}\right)$; во-вторых, (в п. $\left.5^{0}\right)$ дается неожиданно простой ответ на вопрос, поставленный $Г . \Phi$. Вороным в последнем параграфе его последнего, изданного уже посмертно, мемуара [1]. Этот параграф мы, несмотря на ограниченность места в заметке, рискуем привести полностью.

“§118. Рассмотрим две таблицы характеристик граней симплексов, определяющих второй и третий тип примитивных параллелоэдров, мы замечаем, что эти характеристики совпадают для обоих типов и представляются линейными формами

$$
\begin{gathered}
\quad \pm x_{1}, \pm x_{2}, \pm x_{3}, \pm x_{4}, \pm\left(x_{1}-x_{3}\right), \pm\left(x_{1}-x_{4}\right), \pm\left(x_{2}-x_{3}\right), \\
\pm\left(x_{2}-x_{4}\right), \pm\left(x_{3}-x_{4}\right), \pm\left(x_{1}+x_{2}-x_{3}\right), \pm\left(x_{1}+x_{2}-x_{4}\right), \pm\left(x_{1}+x_{2}-x_{3}-x_{4}\right) .
\end{gathered}
$$

Замечательно, что эти линейные формы определяют множество представлений минимума совершенной формы $\varphi_{1}$, определенной равенством (5).

На основании того, что было сказано выше, можно утверждать, что отмеченное совпадение выявляется при рассмотрении характеристик граней всех примитивных параллелоэдров 2,3 и 4 измерений.

Было бы интересно выяснить, является ли это совпадение делом случая или же действительно существует связь между двумя проблемами, кажущимися столь различными: между проблемой однозначного разбиения пространства с помощью вьпуклых конгруэнтных многогранников и проблемой разыскания совершенных положительных квадратичных форм."

$1^{0}$. Теорема 1. Существуют такие числа $1=n_{1}<n_{2}=4<n_{3}=5<\cdots<n_{k}<\ldots$, что в евклидовых пространствах $\mathbf{E}^{n_{k}}$, определены, вообще говоря, не примитивные, ПалЭ (которые мы называем коренными), удовлетворяющие следующим условиям:

1) при каждом натуральном п для любого п-мерного примитивного ПалЭ $\mathscr{P} \subset \mathbf{E}^{n}$ найдется не менее чем $n(n+1) / 2$ коренных ПалЭ $\mathscr{P}_{1}, \mathscr{P}_{2}, \ldots, \mathscr{P}_{\sigma}$, имеющих размерность не превосходящую $n$, и так расположенных в $\mathbf{E}^{n}$, что

$$
\mathscr{P}=\lambda_{1} \mathscr{P}_{1} \oplus \lambda_{2} \mathscr{P}_{2} \oplus \cdots \oplus \lambda_{\sigma} \mathscr{P}_{\sigma},
$$

где через $\oplus$ обозначено векторное сложсение (суммирование по Минковскому), а $\lambda_{1}$, $\lambda_{2}, \ldots, \lambda_{\sigma}-$ положительные числа;

2) каждый $п$-мерный примитивный ПалЭ $\mathscr{P}^{\prime} \subset \mathbf{E}^{n}$ того же типа, что и Я尹, представим (с точностью до движения ПалЭ $\mathscr{P}^{\prime}$ в пространстве $\mathbf{E}^{n}$ и аффинного преобразования всего пространства $\left.\mathbf{E}^{n}\right)$ в виде суммы (*), но быть может с другим набором чисел $\lambda_{1}, \lambda_{2}, \ldots, \lambda_{\sigma}$.

Теорема доказьвается применением теорем из $\S \S 41,69-76,85$ и 86 работы [1].

$2^{0}$. В размерности 1 имеется лишь один ПалЭ- отрезок.

ПримеР 1. Любой $n$-мерный примитивный ПалЭ первого типа [1,] [2] является суммой вида $(*)$ отрезков $\mathscr{P}_{1}, \mathscr{P}_{2}, \ldots, \mathscr{P}_{n(n+1) / 2}$, расположенных в $\mathbf{E}^{n}$ на ребрах произвольного $n$-мерного симплекса. Каждый $n$-мерный не примитивный ПалЭ первого типа [1], [2] является суммой вида $(*)$ части, расположенных указанным способом отрезков. Таким образом, каждый отрезок является коренным одномерным ПалЭ.

(Из [3] следует, что $n(n+1) / 2$ - это максимальное для получения ПалЭ число отрезков, которое здесь возможно использовать.)

$3^{0}$. В размерностях 2 и 3 есть только ПалЭ первого типа: здесь нет коренных ПалЭ. При $n=4$, кроме ПалЭ первого типа, есть примитивные ПалЭ еще двух типов. Опишем построение этих ПалЭ по нашей теореме. Рассмотрим в $\mathbf{E}^{4}$ правильньй 24 -гранник $M_{4}$ (все его грани правильные октаэдры). Этот многогранник $M_{4}$ является ПалЭ (см. также $4^{0}$.)

Работа поддержана РФФИ, грант 97-01-00266. 
Вместе с каждым ребром многогранник $M_{4}$ содержит еще 7 равных и параллельных ему ребер. Выберем из каждой такой восьмерки ребер по одному ребру и обозначим полученное множество через $R_{1}$. (Одному из таких множеств вида $R_{1}$ соответствует, например, множество взятых лишь с плюсом "характеристик" из приведенной цитаты.) С точностью до выбора ребер из восьмерки и вращения многогранника $M_{4}$, в множестве $R_{1}$ имеется толко три вида некомпланарных троек ребер и одна тройка компланарных. Обозначим через $R_{2}$ множество $R_{1}$ без той тройки некомпланарных ребер, в которой каждое ребро составляет с другим угол $60^{\circ}$. Обозначим через $R_{3}$ множество $R_{1}$ без тройки компланарных ребер.

ПримеР 2. Каждый 4-мерный примитивньй ПалЭ второго (третьего) типа Я尹P представим (с точностью до движения ПалЭ $\mathscr{P}$ в пространстве $\mathbf{E}^{4}$ и афффинного преобразования всего пространства $\left.\mathbf{E}^{4}\right)$ в виде суммы $(*)$, где через $\mathscr{P}_{1}$ обозначен многогранник $M_{4}$, а через $\mathscr{P}_{i}$, где $i=2,3, \ldots, 10,-$ отрезки из множества $R_{2}\left(R_{3}\right)$.

Из сказанного следует, что многогранник $M_{4}$ (и любой многогранник ему аффинно эквивалентный) является коренным 4-мерным ПалЭ, причем с точностью до афффинной эквивалентности единственным

Напомним [2], что при $n \leqslant 4$ все ПалЭ, а не толко примитивные, суть аффинные образы областей Вороного-Дирихле решеток. Поэтому, чтобы завершить наше предъявляемое впервые полное элементарное и наглядное описание всех 4-мерных ПалЭ, см. также [4], нам осталось сделать два замечания. (1) ПалЭ под номером 19 из статьи [2] получаются как суммы вида $(*)$ всех отрезков множества $R_{3}$. (2) Все осталшные, не упомянутые в $2^{0}$ и $3^{0}, 4$-мерные ПалЭ могут быть получены как суммы вида $(*)$ многогранника $M_{4}$ и части отрезков из множества $R_{3}$.

$4^{0}$. Рассмотрим вторую совершенную форму $\left(V_{n}\right.$ по Коркину и Золотареву [3], $\varphi_{1}^{(n)}$ по Вороному [1], или $D_{n}$ по Коксетеру [5]).

Область Вороного-Дирихле $M_{n}$ решетки, соответствующей форме $V_{n}$, с точностью до подобия, может быть описанной нижеследующим образом. Многогранник $M_{n}$ - это $n$-мерньй куб $Q_{n} \subset \mathbf{E}^{n}$ с построенной над каждой его $(n-1)$-мерной гранью равнореберной пирамидой с высотой, равной половине ребра куба. (Отметим, что такого описания многогранника $M_{n}$ мы нигде не встречали.)

Многогранник $M_{n}$ и при всех $n>4$ является коренньп ПалЭ (при $n=4$ мы дали для $M_{4}$ другое описание). Часть из остальных коренных ПалЭ, способных вместе с многогранником $M_{n}$ задавать примитивные ПалЭ - это $n-1$ произвольных попарно не коллинеарных ребер куба $Q_{n}$.

Этот пример позволяет также утверждать в теореме 1 , что $n_{k}=k+2$ при всех $k>1$.

$5^{0}$. Отметим, что у многогранника $M_{n}$ можно выбрать $2^{n-1}+n$ попарно не коллинеарных ребер, т.е. ему соответствует $2^{n}+2 n$ различных характеристик - это число превосходит максимальное количество представлений арифометического минимума положительно определенной формы (или оценку контактного числа) при всех $4<n \leq 24$ и всех достаточно болшших $n$ (см., например, [6]). Следовательно, множество этих характеристик не может быть частью множества представлений минимума никакой положительно определенной квадратичной формы от указанного числа $n$ переменных.

Таким образом, замеченное $Г$. $\Phi$.Вороньм при $n \leqslant 4$ совпадение при болших $n$, вообще говоря, отсутствует.

\section{СПИСОК ЛИТЕРАТУРЫ}

[1] Вороной Г.Ф. Исследования о примитивных паралеллоэдрах // Собр. соч.. Т. 2. Киев: Изд-во АН УССР, 1952. С. 239-368 (Оригинал в J. reine und angew. Math., 1908, v. 134, P. 198-287, 1909, vol. 136, P. 67-179). [2] B.Delaunay Sur la partition régulière l'espace a 4 dimensions // Изв. АН, ОФМН 1929. № 1. С. 79-110; № 2, Р. 147-164. [3] Korkine A., Zolotareff G. Sur les forme quadratiques positives // Math. Ann. 1877. V. 11. P. 242-292 (См. также Е.И.Золотарев, Полное собр. соч., вып.1, Изд-во АН. 1931, С. 375-434.). [4] С.С.Рышков, Л.В.Краснова, Н.С.Шугарова K вопросу о классификации типов четырехмерных непримитивных параллелоэдров Дирихле // Международная конференция "Современные проблемы теории чисел".. Россия, Тула: Тезисы докладов, 1993. [5] Coxeter, H. S. M. Extreme forms // Can. J. of Math 1951. V. 3. Р. 391-441. [6] Конвей Дж., Слоэн Н. Упаковки шаров, решетки и группы. Москва: Мир, 1990. 